Revista Brasil. Bot., V.28, n.4, p.745-754, out.-dez. 2005

\title{
Morfologia e anatomia do fruto e da semente em desenvolvimento de Pilocarpus pennatifolius Lem. (Rutaceae)
}

\author{
ANDREZA DE SOUZA ${ }^{1}$, KÁTHIA S. MATHIAS MOURÃO ${ }^{1,2}$ e LUIZ ANTONIO DE SOUZA ${ }^{1}$
}

(recebido: 5 de agosto de 2004; aceito: 20 de outubro de 2005)

\begin{abstract}
Morphology and anatomy of the fruit and seed in development of Pilocarpus pennatifolius Lem. (Rutaceae)). The developing fruit and seed of Pilocarpus pennatifolius were investigated. The fruit is schizocarpic with one to five mericarps. The endocarp is sclerified and originates from the inner epidermis of the ovary. The endocarp participates actively in the dehiscence of the fruit. The seed is campylotropus, bitegmic and mesotestal.
\end{abstract}

Key words - fruit development, Pilocarpus pennatifolius, Rutaceae, seed development

RESUMO - (Morfologia e anatomia do fruto e da semente em desenvolvimento de Pilocarpus pennatifolius Lem. (Rutaceae)). O fruto e a semente em desenvolvimento de Pilocarpus pennatifolius foram investigados. O fruto é um esquizocarpo, com um a cinco mericarpos. O endocarpo é esclerenquimático e se origina da ação meristemática da epiderme interna do ovário. O endocarpo participa ativamente do processo de deiscência do fruto. A semente é campilótropa, bitegumentada e mesotestal.

Palavras-chave - fruto, Pilocarpus pennatifolius, Rutaceae, semente

\section{Introdução}

A família Rutaceae conta com cerca de 150 gêneros e 1.600 espécies, que estão distribuídas nos trópicos, subtrópicos e regiões temperadas do hemisfério sul. No Brasil está representada por mais ou menos 29 gêneros e 182 espécies (Barroso et al. 1984).

Dentre as espécies dessa família destaca-se Pilocarpus pennatifolius Lem., incluída na subfamília Rutoideae, tribo Cuspareae, conhecida popularmente como "jaborandi", cuja ocorrência é registrada desde Pernambuco até Santa Catarina. É uma planta medicinal preciosa, diaforética, diurética e sialagoga, que contém o princípio ativo pilocarpina. O alcalóide pilocarpina tem ação fisiológica sobre o olho humano, tonifica as glândulas sudoríparas e salivares e têm influência sobre os brônquios, a mucosa nasal e as glândulas lacrimais (Pio-Corrêa 1978).

Pilocarpus pennatifolius é uma arvoreta freqüente nos remanescentes florestais urbanos da cidade de Maringá, Paraná, Brasil. Apresenta folhas compostas, coriáceas, imparapinadas e folíolos grandes. As flores são pequenas, de coloração rosada e dispostas em rácemos longos (Cowan \& Smith 1973).

A literatura botânica registra escassos estudos sobre anatomia de órgãos reprodutivos em Rutaceae. Com

\footnotetext{
1. Universidade Estadual de Maringá, Departamento de Biologia, Av. Colombo 5790, 87020-900 Maringá, PR, Brasil.

2._Autor para correspondência: ksmmourao@uem.br
}

relação ao desenvolvimento do pericarpo, não foram encontrados estudos anatômicos detalhados. Entretanto, Beltrati (1991), apesar de não ter realizado estudo anatômico do fruto de Esenbeckia febrifuga A. Juss., afirma que o endocarpo seco e lignificado participa da ejeção das sementes. Em Pilocarpus pennatifolius, à semelhança do que ocorre em E. febrifuga e em outras espécies de Rutaceae, as sementes são ejetadas do fruto na maturidade, não se sabendo exatamente qual ou quais tecidos participam deste processo.

Barroso et al. (1999) afirmam que nas flores de Rutaceae os carpelos estão pouco aderidos entre si e que com o desenvolvimento do fruto este se torna múltiplo, como se verifica em espécies dos gêneros Pilocarpus Vahl., Zanthoxylum L., Galipea Aubl., Spiranthera A. St.-Hil., Almeidea A. St.-Hil., Monnieria L., entre outros. Por outro lado, Souza et al. (2003) descrevem o gineceu de Pilocarpus pennatifolius como parcialmente apocárpico, sendo os carpelos separados nas regiões média a superior do ovário, e afirmam que durante o desenvolvimento do fruto, com a senescência do estigma e estilete, acentua-se a separação dos carpelos.

Com relação à semente, destaca-se o extenso trabalho sobre anatomia de sementes de dicotiledôneas, realizado por Corner (1976). Este autor descreveu as sementes de 17 espécies e de um gênero de Rutaceae, incluindo Pilocarpus racemosus Vahl. Destacou como características da família a predominância da testa como camada mecânica, tanto da exo como da mesotesta, a falta de diferenciação da endotesta e a constituição 
traqueoidal do tégmen. Estudos detalhados sobre o desenvolvimento do óvulo e da testa em Rutaceae também foram realizados por Boesewinkel $(1977,1978)$ em Ruta L., Zanthoxylum L., Skimmia Thunb., Poncirus Raf., Citrus L., Fortunella Swingle, Citropsis Swingle \& Kellerman e Murraya Koen. ex L., e por Boesewinkel \& Bouman (1978), em Glycosmis arborea (Roxb.) DC. Mais recentemente, Beltrari (1991) descreveu a estrutura da semente de Esenbeckia febrifuga. Todos estes estudos destacam a grande diversidade estrutural das sementes nesta família.

A grande escassez de estudos ontogênicos detalhados dos frutos e sementes de angiospermas tem dificultado a classificação dos tipos de frutos e também das estruturas que envolvem as sementes. Vários autores, tais como Beltrati (1978) e Groth et al. (1983) utilizam características das sementes com finalidades taxonômicas, visto que são pouco alteráveis frente às variações ambientais. Segundo estes autores, as características da semente são úteis para identificação de gêneros e, possivelmente, espécies.

Em vista do exposto, pretende-se, no presente trabalho, fornecer dados morfológicos e anatômicos a respeito dos frutos e sementes em desenvolvimento de Pilocarpus pennatifolius de modo a ampliar o conhecimento sobre a estrutura destes órgãos em Rutaceae e sobre a biologia desta espécie, contribuindo assim, com futuros estudos taxonômicos e ecológicos do grupo.

\section{Material e métodos}

O material utilizado constou de botões florais, flores (figura 1) e frutos (figura 2) de Pilocarpus pennatifolius Lem., em diferentes estádios de desenvolvimento, coletados na mata do Horto Florestal Dr. Luiz Teixeira Mendes, em Maringá, Paraná, Brasil.

Material estudado: Pilocarpus pennatifolius Lem.: BRASIL. Paraná: Maringá, Horto Florestal Dr. Luiz Teixeira Mendes, 22-IX-1999, A. de Souza 1 (HUM 4764); BRASIL. Paraná: Maringá, Horto Florestal Dr. Luiz Teixeira Mendes, 16-VII-1999, A. de Souza 2 (HUM 4765).

Os estudos morfológicos e anatômicos foram realizados em material fresco e fixado. As características morfológicas dos frutos e sementes foram descritas e ilustradas utilizando-se material proveniente de três indivíduos. A nomenclatura para descrever as formas dos frutos e sementes foi baseada em Radford et al. (1974).

Para determinação das dimensões (comprimento e maior diâmetro) dos mericarpos e sementes foi utilizado um paquímetro. Determinou-se, também, o número de mericarpos por fruto. Para cada uma das variáveis obtidas foi
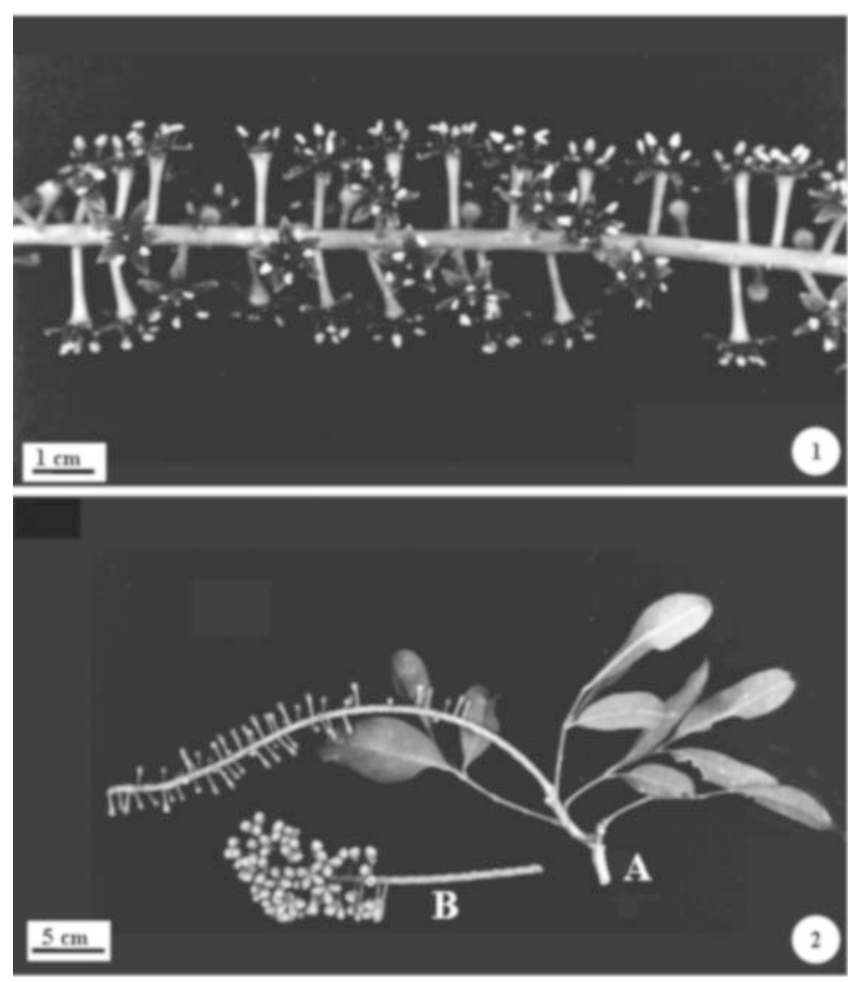

Figuras 1-2. Aspecto morfológico da inflorescência e do fruto de P. pennatifolius. 1. Parte do cacho. 2. Frutos jovens (A) e em pré-deiscência (B).

Figures 1-2. Morphological aspect of the inflorescence and fruit of P. pennatifolius. 1. Segment of the racem. 2. Young fruits (A) and in pre-dehiscence phase (B).

calculada a média aritmética, o desvio-padrão e a amplitude de variação.

Para a fixação de material visando estudos morfológicos e anatômicos foi utilizado FAA (Johansen 1940). A conservação desse material foi feita em etanol $70 \%$ (Jensen 1962). A descrição anatômica foi feita a partir da análise de laminário semipermanente e permanente confeccionado com cortes transversais e longitudinais do pericarpo e das sementes, em desenvolvimento. Este laminário e as ilustrações foram feitas de acordo com metodologia descrita por Mourão \& Beltrati (1995).

Cortes de material fresco foram submetidos a testes microquímicos, utilizando-se: solução de Sudan III e Sudan IV, para substância de caráter lipídico (Rawlins \& Takahashi 1952); solução aquosa de cloreto férrico a $10 \%$, adicionada de pequena porção de carbonato de cálcio, e fixação em solução aquosa contendo $3 \%$ a $5 \%$ de formalina a $10 \%$ de sulfato ferroso por 24 a 48 horas para localização de compostos fenólicos (Johansen 1940); floroglucinol acidificado (Johansen 1940) para identificação de lignina; reagente de lugol para localização de amido (Jensen 1962).

Utilizaram-se também na confecção de lâminas permanentes materiais incluídos em "Historesin" da marca 
Leica, seguindo-se as recomendações do fabricante. Estas lâminas foram coradas com azul de toluidina $\mathrm{O}$ (O'Brien et al. 1964) e montadas em Permount. A espessura dos cortes feitos em micrótomo variou de 10 a $15 \mu \mathrm{m}$.

A terminologia adotada para definir as camadas do pericarpo está de acordo com Roth (1977), a classificação do tipo de fruto de acordo com Spjut (1994) e a nomenclatura utilizada na descrição das sementes foi a definida por Corner (1976), modificada por Schmid (1986).

\section{Resultados e Discussão}

Desenvolvimento do fruto - O ovário de Pilocarpus pennatifolius Lem. é súpero, pentacarpelar, pentaloculado, apresentando dois óvulos por lóculo de placentação axilar. Os carpelos estão parcialmente unidos a partir da região mediana do ovário, separando-se completamente no ápice e unindo-se no estilete (figuras 11-13). Souza et al. (2003) analisaram a vascularização floral do gineceu dessa espécie e constataram que a fusão dos carpelos na base do ovário não acarreta qualquer efeito sobre seus feixes vasculares marginais ou ventrais e dorsal. De acordo ainda com esses autores, tais feixes vasculares mantêm-se individualizados, sem qualquer fusão dentro do mesmo carpelo ou com feixes de carpelos contíguos, o que favorece a interpretação de apocarpia em Pilocarpus pennatifolius.

A estrutura do ovário (figuras 11-13, 15, 16) de Pilocarpus pennatifolius, já descrita em detalhe por Souza et al. (2003), revela a presença de epiderme externa ou dorsal unisseriada com tricomas glandulares, mesofilo parenquimático com conspícuas cavidades secretoras e epiderme interna ou ventral multisseriada com células de formato tabular e paredes finas. A natureza multisseriada da epiderme interna ovariana se deve à ação meristemática que se instala nesse tecido epidérmico ainda no estágio floral de pré-antese. No ovário ocorrem ainda células contendo substâncias fenólicas esparsas por todo o mesofilo e ao redor dos feixes vasculares.

O ovário de Pilocarpus pennatifolius apresenta diferentes estruturas secretoras, como tricomas glandulares pluricelulares na epiderme externa, amplas cavidades secretoras no mesofilo, e nectários localizados na base ovariana. Essas estruturas, investigadas por Souza et al. (2003), é característica comum à maioria das Rutaceae, conforme descrevem Metcalfe \& Chalk (1972) e Cronquist (1981).

A definição de exo, meso e endocarpo varia de acordo com o ponto de vista de cada autor. A maioria dos autores prefere usar os termo exo e endocarpo em sentido amplo, que podem incluir, além da epiderme externa e interna, respectivamente, também alguns tecidos subepidérmicos (Roth 1977). De acordo com Fahn (1990), a separação do pericarpo em três camadas distintas facilita a descrição anatômica, mas elas não representam tecidos separados quanto à sua origem. A análise do fruto em desenvolvimento de Pilocarpus pennatifolius levou em consideração a origem de todos os tecidos pericárpicos, desde a fase de ovário de flores e botões florais. Adotou-se assim, no estudo estrutural do pericarpo dessa espécie, a terminologia de Souza (2003), que se baseia na ontogênese do exocarpo, mesocarpo e endocarpo, referidos como provenientes da epiderme externa ou dorsal, mesofilo, e epiderme interna ou ventral do ovário, respectivamente.

Em Pilocarpus pennatifolius o exocarpo, derivado somente da epiderme externa do ovário, caracteriza-se por apresentar células cúbicas, as quais perdem o aspecto papiloso durante o desenvolvimento do fruto (figuras 15, 17, 19).

O mesocarpo de Pilocarpus pennatifolius, derivado do mesofilo ovariano (figuras 15, 16), é constituído por hipoderme esclerificada, cinco a seis camadas de tecido colenquimatoso abaixo da hipoderme e por tecido parenquimático (figura 19). Nesse parênquima observam-se cavidades secretoras de óleos e feixes vasculares colaterais acompanhados externamente por fibras (figura 20).

O endocarpo de Pilocarpus pennatifolius é derivado da epiderme interna do ovário. Essa epiderme assume atividade de meristema ventral, ainda em pré-antese, e torna-se multisseriada (figuras 16, 21). Esta descrição também é ressaltada por Souza et al. (2003).

As células desta epiderme multisseriada alongam-se tangencialmente no fruto jovem (figura 18), e diferenciam-se em fibras no fruto maduro. Os vários estratos de fibras do endocarpo se separam como unidade do restante do pericarpo na maturidade (figuras 8,14 , 20), permanecendo presos ao funículo endurecido e cordiforme até a ejeção da semente (figuras 9, 10). Com a ejeção, o endocarpo e o funículo se separam da semente e também são liberados do fruto.

Apesar de não ter realizado estudo anatômico do desenvolvimento do pericarpo em Esenbeckia febrifuga A. Juss., Beltrati (1991), descreveu que no fruto desta espécie o endocarpo é o responsável pela autocoria, à semelhança de Pilocarpus pennatifolius. Este resultado está de acordo com a afirmação de Souza et al. (2003), de que o meristema instalado precocemente na epiderme ventral do ovário deve provavelmente contribuir no desenvolvimento do aparelho de deiscência do fruto. A formação do endocarpo a partir da epiderme interna da 
parede ovariana é grandemente enfatizada como característica comum em Rutaceae, e é considerada como atributo dessa família (Hartl apud Boesewinkel 1977).

Segundo Roth (1977), os mecanismos de deiscência estão baseados, em geral, em movimentos de tecidos vivos ou mortos. Dois tipos fundamentais de mecanismos de deiscência podem ocorrer: o higroscópico e o de turgor. Os higroscópicos dependem, geralmente, do encolhimento ou distensão de paredes de células mortas, enquanto o de turgor funciona com células vivas, de paredes elásticas.

Na deiscência higroscópica, Roth (1977) considera dois tipos: o xerocásico e o higrocásico. As forças atuantes dos movimentos higrocásicos repousam no encharcamento de água, que ocasiona intumescimento das paredes celulares, desenvolvendo-se pressões de distensão, ao passo que no xerocásico, pela perda de água resulta no encolhimento das paredes celulares. No mecanismo higrocásico, a fonte de energia está no poder de intumescimento das células, enquanto no xerocásico a energia é desenvolvida por tensão de forças. Para produzir a curvatura de certas partes do pericarpo há duas camadas que atuam antagonicamente. Estas podem apresentar estrutura diferente de seus elementos, geralmente constituídas de fibras, que podem estar dispostas em direções diferentes, de modo que as células de uma camada cruzam com as de outra camada.

Em Pilocarpus pennatifolius, sem dúvida o mecanismo é higroscópico xerocásico, uma vez que o pericarpo torna-se seco com a maturidade. O fruto abre-se ao longo da sutura ventral da folha carpelar em direção ao seu ápice, observando-se uma região de fraqueza mecânica, margeada internamente por feixes vasculares e, externamente, por hipoderme esclerenquimática. Esta região é representada pela linha de deiscência (figura 14), constituída por poucas fileiras de células parenquimáticas de paredes delgadas em relação às do parênquima do restante do mesocarpo.

Ainda em Pilocarpus pennatifolius, além das fibras que acompanham os feixes vasculares e da hipoderme esclerificada, as fibras endocárpicas e o tecido funicular esclerificado participam gerando forças de tensão para que o fruto se abra pela linha de deiscência. A ejeção das sementes se deve às forças de tensão produzidas pelas fibras endocárpicas que se encontram dispostas em diferentes direções, ambas oblíquas (figura 20). Este aspecto é observado no endocarpo que é liberado juntamente com a semente e encontrado torcido no solo. Pode ser comprovado quando os frutos imaturos são trazidos ao laboratório e deixados em local seco por algum tempo, completando-se a maturação. As forças de tensão são tão grandes que se escuta um ruído quando as sementes são ejetadas do fruto.

A apocarpia em Pilocarpus pennatifolius, constatada por Souza et al. (2003) em estudo anatômico e de vascularização da flor, acentua-se durante o desenvolvimento do fruto. $O$ fruto maduro dessa espécie se enquadra no tipo esquizocarpo, subtipo Coccarium, caracterizado por Spjut (1994) como derivado de um gineceu esquizocárpico que origina frutículos que se abrem ao longo da sutura ventral. Este autor menciona este tipo de fruto em outras Rutaceae, como Esenbeckia flava Brandegee, E. panamensis T.S. Elias, Zanthoxylum setulosum P. Wilson, Z. arborescens Rose e Z. martinicense DC.

Por outro lado, é importante destacar que Barroso et al. (1999) consideram o fruto de Pilocarpus Vahl., Zanthoxylum L., Galipea Aubl., Spiranthera A. St.-Hil., Almeidea A. St.-Hil. e Monnieria L. como múltiplo, caracterizado, nesse caso, como aquele que deriva de gineceu apocárpico ou apocarpóide, com carpelos levemente unidos na base.

Estudos mais detalhados de anatomia floral e da ontogênese do fruto podem auxiliar no entendimento da verdadeira origem dos frutos das angiospermas e, com certeza, contribuírem para a uniformização da classificação. É conveniente, portanto, definir-se na metodologia qual proposta seguiu-se para classificar o fruto da espécie objeto de estudo.

Em Pilocarpus pennatifolius, o esquizocarpo, de coloração marrom após a maturação, apresenta número variável de mericarpos unicarpelares e unisseminados (figuras 2, 3-7), sendo estes de tamanho variável. Essa variação se deve ao aborto de um a quatro mericarpos, que se verifica ao longo do desenvolvimento do fruto (tabela 1).

Desenvolvimento da semente - Os óvulos de Pilocarpus pennatifolius são hemítropos, bitegumentados e crassinucelados (Souza et al. 2003). O tegumento externo ovular apresenta de duas a quatro camadas de células e o tegumento interno duas a três camadas. São observadas as cutículas externa, mediana (entre os dois tegumentos) e a interna (entre o tegumento interno e o nucelo). $\mathrm{O}$ canal micropilar é formado pela exóstoma e pela endóstoma, sendo as aberturas não coincidentes (micrópila em zig-zag). Na região calazal observam-se células com paredes delgadas de conteúdo fenólico e que constituem a hipóstase. $\mathrm{O}$ traço rafeal percorre o funículo e a região rafeal, chegando somente até a calaza (figura 21).

Apesar dos óvulos das Rutaceae e, especialmente de Pilocarpus terem sido descritos por Netolitzky (1926) 
e Corner (1976) como anátropos, em Pilocarpus pennatifolius o óvulo é hemítropo, à semelhança do que ocorre em Esenbeckia febrifuga (Beltrati 1991). Em ambas as espécies, o óvulo apresenta tendência à campilotropia. Esta constatação está de acordo com a afirmação de Boesewinkel (1977).
O termo hipóstase foi proposto por Van Tieghen \& Johansen (apud Tilton 1980) para designar todos os tipos de modificações da calaza e/ou da região calazal do óvulo. Tilton (1980) após extensa discussão, sugere a seguinte definição para hipóstase: um grupo de células modificadas com paredes geralmente lignificadas
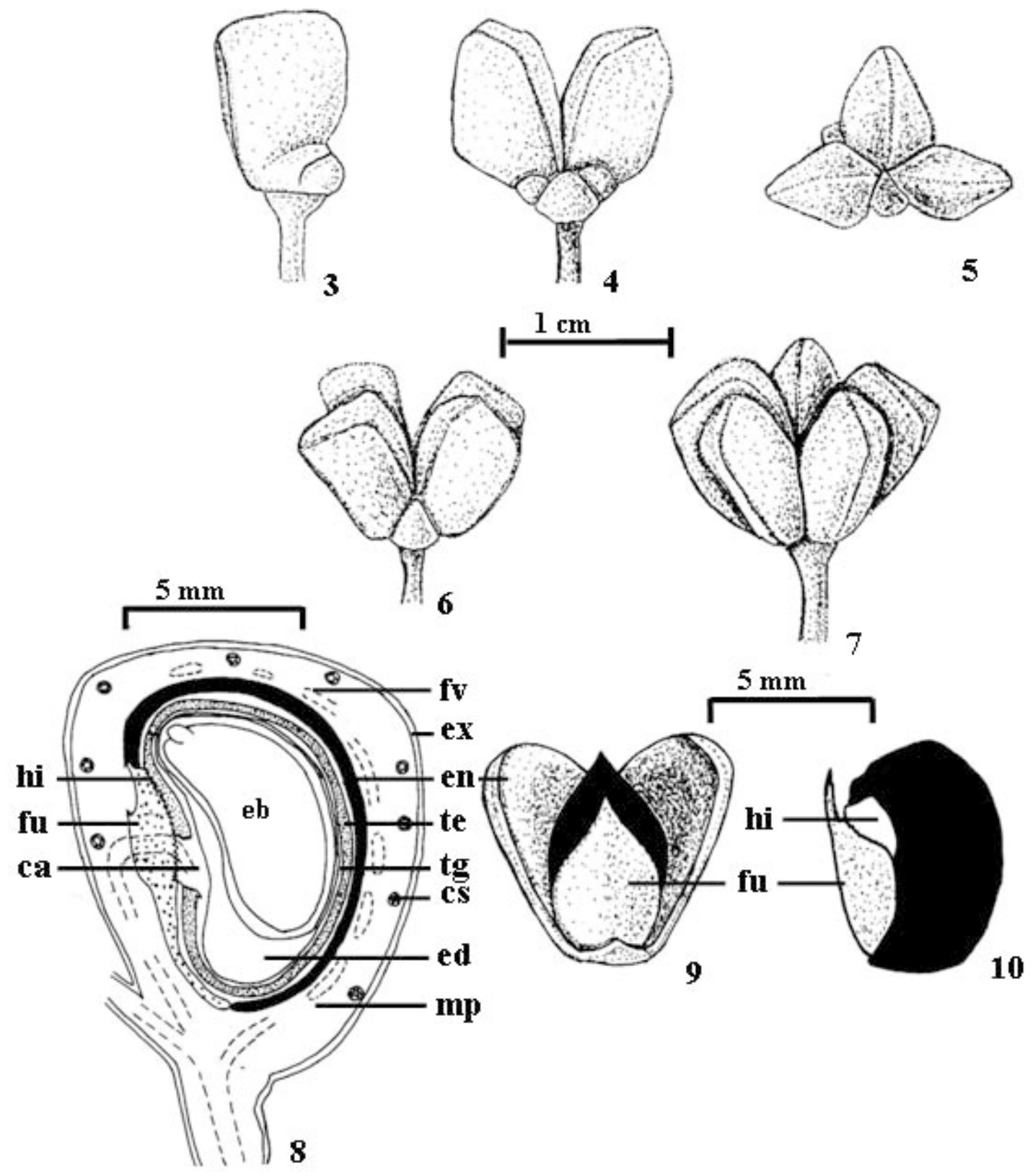

Figuras 3-10. Frutos imaturos e semente de P. pennatifolius. 3-7. Frutos imaturos com 1; 2; 3; 4 e 5 mericarpos; respectivamente. 8. Fruto imaturo em secção longitudinal. 9-10. Semente aderida ao endocarpo em vista hilar e lateral; respectivamente. $(\mathrm{ca}=$ calaza; $\mathrm{cs}=$ cavidade secretora; $\mathrm{eb}=$ embrião; ed $=$ endosperma; $\mathrm{en}=$ endocarpo; ex = exocarpo; $\mathrm{fv}=$ feixe vascular; fu = funículo; hi = hilo; $\mathrm{mp}=$ mesocarpo; te = testa; $\operatorname{tg}=$ tégmen $)$.

Figures 3-10. Immature fruits and seed of $P$. pennatifolius. 3-7. Immature fruits with 1;2;3; 4 and 5 mericarps; respectively. 8. Immature fruit in longitudinal section. 9-10. Seed attached to the endocarp in hilar and lateral view; respectively ( $\mathrm{ca}=$ chalaza; $\mathrm{cs}=$ secretory cavity; $\mathrm{eb}=$ embryo; $\mathrm{ed}=$ endosperm; em = endocarp; $\mathrm{ex}=$ exocarp; $\mathrm{fv}=$ vascular bundle; $\mathrm{fu}=$ funicle; hi = hilum; $\mathrm{mp}=$ mesocarp; te = testa; $\operatorname{tg}=$ tegmen $)$. 
Tabela 1. Número de mericarpos por fruto $(\mathrm{N}=150)$ e dimensões do mericarpo $(\mathrm{N}=60)$ de Pilocarpus pennatifolius. Table 1. Number of mericarps per fruit $(\mathrm{N}=150)$ and mericarp measures $(\mathrm{N}=60)$ of Pilocarpus pennatifolius.

\begin{tabular}{lccc}
\hline & Número & \multicolumn{2}{c}{ Dimensões } \\
\cline { 3 - 4 } & de mericarpos & $\begin{array}{c}\text { Comprimento } \\
(\mathrm{mm})\end{array}$ & $\begin{array}{c}\text { Diâmetro } \\
\text { (valor máximo) } \\
(\mathrm{mm})\end{array}$ \\
\hline Média & 3,30 & 10,82 & 8,09 \\
Desvio padrão & 6,45 & 1,00 & 1,12 \\
Amplitude de variação & $1-5$ & $9,6-13$ & $6,7-10$ \\
\hline
\end{tabular}

presentes na região calazal do óvulo mas que pode envolver uma parte do megagametófito e estender-se parcialmente na região micropilar.

As células com conteúdo fenólico presentes na região calazal do óvulo de Pilocarpus pennatifolius enquadram-se na definição de hipóstase proposta por Van Tieghen \& Johansen (apud Tilton 1980) e Tilton (1980).
Na região situada entre o funículo e a exóstoma do óvulo de Pilocarpus pennatifolius ocorre uma proliferação de células, revestida por epiderme papilosa, constituindo um obturador, que posteriormente desaparece. Boesewinkel (1977) relata a presença de obturador em Ruta graveolens L. e Zanthoxylum simulans Hance, Boesewinkel \& Bouman (1978) em Glycosmis aborea (Roxb.) DC. e Beltrati (1991) em
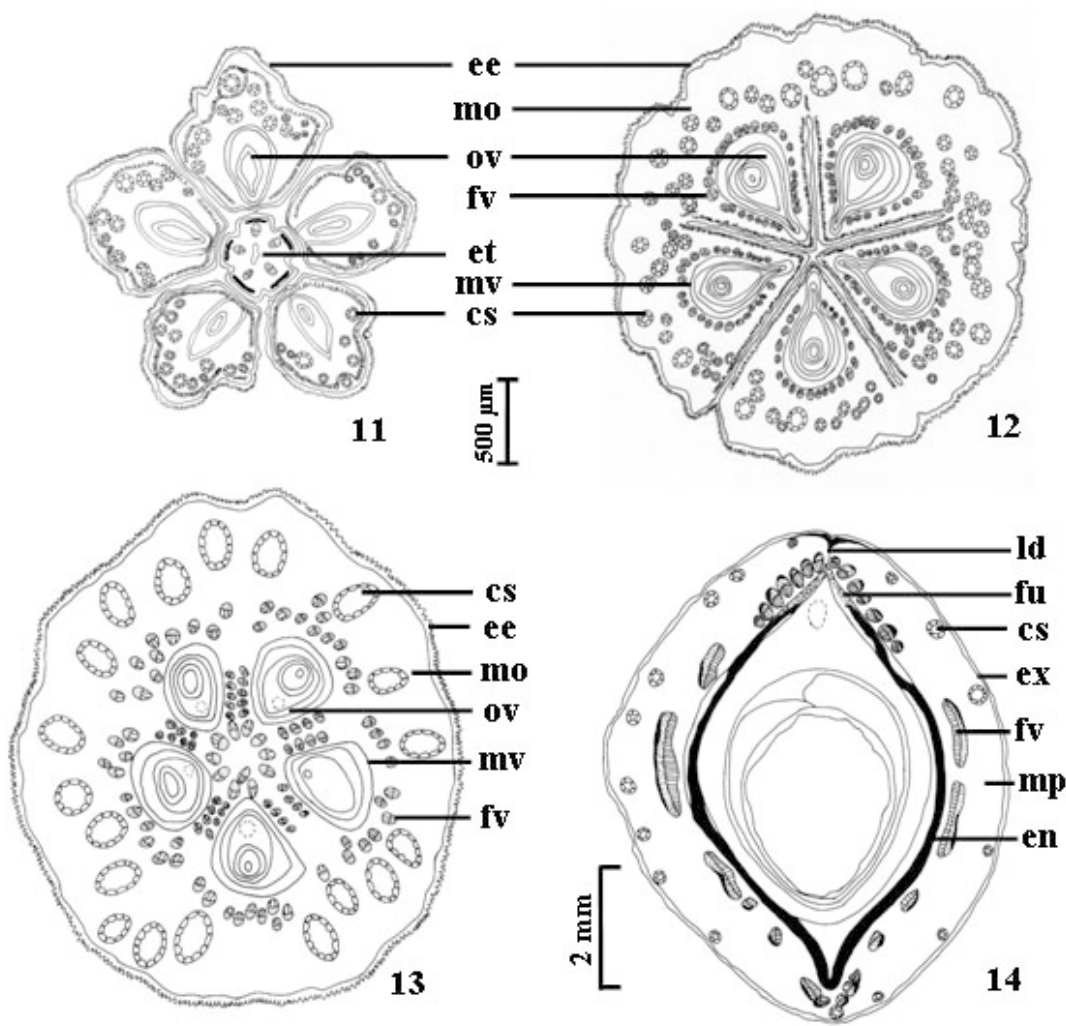

Figuras 11-14. Secções transversais do ovário e fruto de P. pennatifolius. 11-13. Ovário - ápice; meio e base; respectivamente. 14. Fruto maduro (cs = cavidade secretora; ee = epiderme externa; en = endocarpo; et = estilete; ex = exocarpo; fu = funículo; $\mathrm{fv}=$ feixe vascular; $\mathrm{ld}=$ linha de deiscência $; \mathrm{mo}=$ mesofilo ovariano; $\mathrm{mp}=$ mesocarpo; $\mathrm{mv}=$ meristema ventral; ov = óvulo .

Figures 11-14: Ovary and fruit in cross-sections of $P$. pennatifolius. 11-13. Ovary apical; medium and basal region; respectively. 14. Mature fruit ( $c s=$ secretory cavity; ee = outer epidermis; en = endocarp; et = style; $\mathrm{ex}=$ exocarp; fu = funicle; fv = vascular bundle; $\mathrm{ld}=$ dehiscence line; $\mathrm{mo}=$ ovarian mesophyll; $\mathrm{mp}=$ mesocarp; $\mathrm{mv}=$ ventral meristem; ov = ovule). 

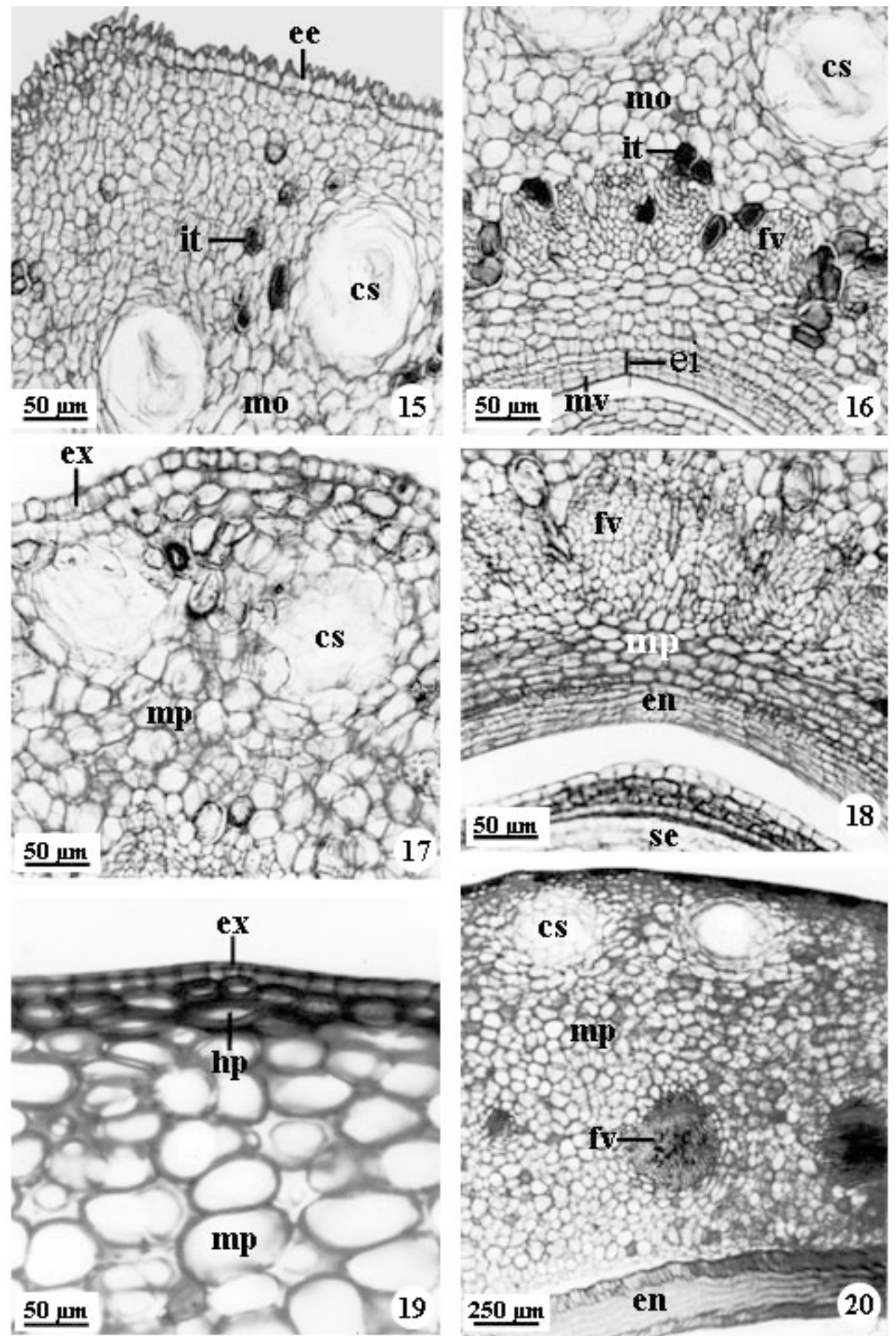

Figuras 15-20. Detalhes de secções transversais do ovário e fruto em desenvolvimento de P. pennatifolius. 15-16. Ovário. 17-18. Fruto jovem. 19-20. Fruto imaturo ( $\mathrm{cs}=$ cavidade secretora; ee = epiderme externa; ei = epiderme interna; en = endocarpo; ex = exocarpo; $\mathrm{fv}=$ feixe vascular; $\mathrm{hp}=$ hipoderme; it = idioblasto tanífero; $\mathrm{mo}=$ mesofilo ovariano; $\mathrm{mp}=$ mesocarpo $; \mathrm{mv}=$ meristema ventral; se $=$ semente) .

Figures 15-20. Details of the ovary and developing fruit in cross-sections of P. pennatifolius. 15-16. Ovary. 17-18. Young fruit. 19-20. Imature fruit (cs = secretory cavity; ee = outer epidermis; ei $=$ inner epidermis; en = endocarp; ex = exocarp; $\mathrm{fv}=$ vascular bundle $\mathrm{hp}=$ hypodermis $;$ it $=$ tannin idioblast $; \mathrm{mo}=$ ovarian mesophyll $; \mathrm{mp}=$ mesocarp $; \mathrm{mv}=$ ventral meristem; se $=$ seed $)$. 
Esenbeckia febrifuga. De acordo com Maheshwari (1978), o obturador, que tem conexão com o tecido transmissor, facilita a entrada do tubo polínico no óvulo. Estudos de embriologia e de biologia floral poderiam confirmar se esta proliferação de células tem a função de obturador em Pilocarpus pennatifolius.

As sementes maduras de Pilocarpus pennatifolius são reniformes, tem dimensões variáveis (tabela 2), e apresentam tegumento de coloração negra (figuras 8-10). O hilo é ovalado e de coloração branca (figura 10). Apresentam ainda pouco endosperma na

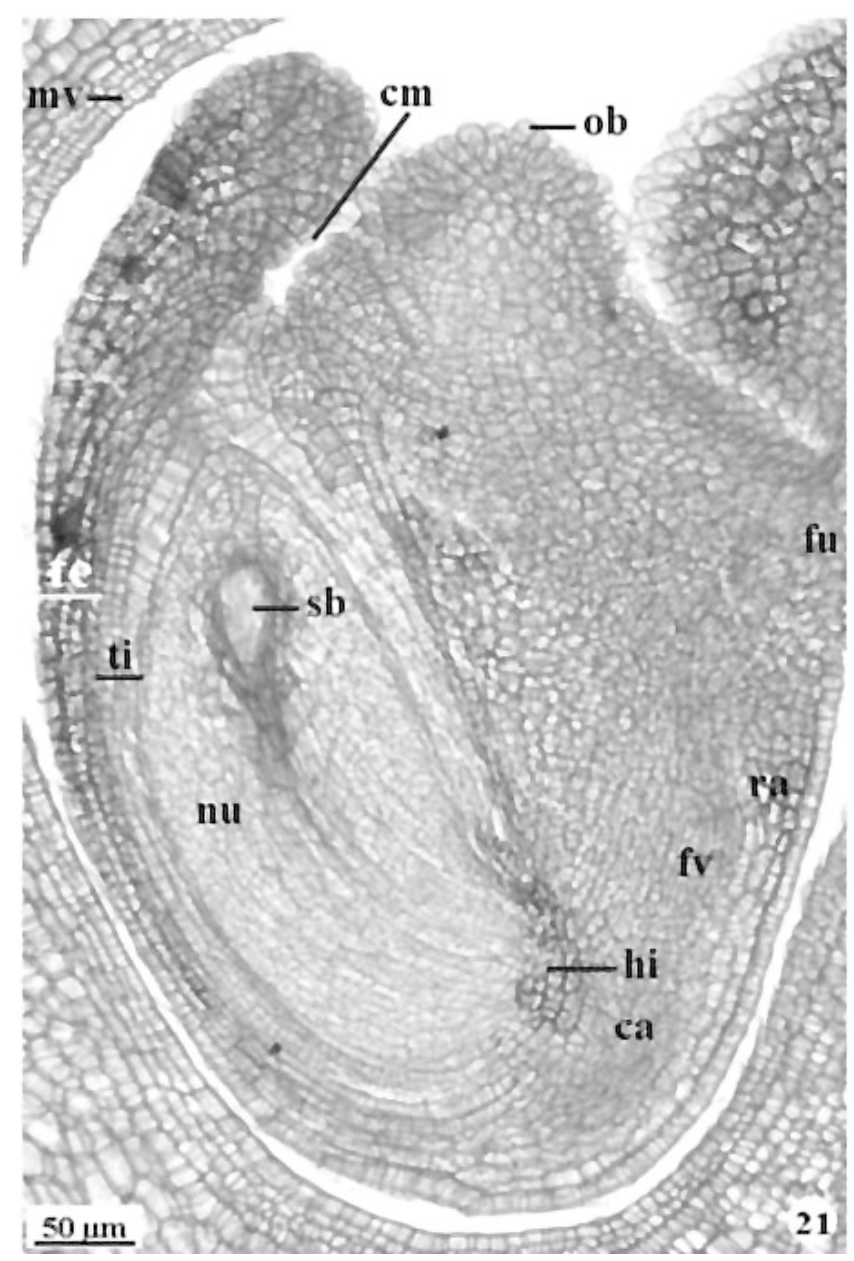

Figura 21. Óvulo em secção longitudinal de P. pennatifolius (ca = calaza $; \mathrm{cm}=$ canal micropilar; $\mathrm{fv}=$ traço vascular rafeal; $\mathrm{fu}=$ funículo; hy = hipóstase $; \mathrm{mv}=$ meristema ventral; $\mathrm{nu}=$ nucelo; $\mathrm{ob}=$ obturador; $\mathrm{ra}=$ rafe; $\mathrm{sb}=$ saco embrionário; te $=$ tegumento externo; $\mathrm{ti}=$ tegumento interno).

Figure 21. Ovule in longitudinal section of $P$. pennatifolius ( $\mathrm{ca}=$ chalaza $; \mathrm{cm}=$ micropylar channel $; \mathrm{fv}=$ rapheal bundle; $\mathrm{fu}=$ funicle; hy = hypostase $\mathrm{mv}=$ ventral meristem; $\mathrm{nu}=$ nucellus; $\mathrm{ob}=$ obturator; $\mathrm{ra}=$ raphe; $\mathrm{sb}=$ embryonic sac; te = outer integument; $\mathrm{ti}=$ inner integument).
Tabela 2. Dimensões da semente de Pilocarpus pennatifolius $(\mathrm{N}=60)$.

Table 2. Seed measures of Pilocarpus pennatifolius $(\mathrm{N}=60)$.

\begin{tabular}{lcc}
\hline & $\begin{array}{c}\text { Comprimento } \\
(\mathrm{mm})\end{array}$ & $\begin{array}{c}\text { Diâmetro } \\
\text { (valor máximo) } \\
(\mathrm{mm})\end{array}$ \\
\hline Média & 8,36 & 4,88 \\
Desvio Padrão & 0,54 & 0,79 \\
Amplitude de variação & $7,60-9,60$ & $3,90-9,90$ \\
\hline
\end{tabular}

maturidade, são bitegumentadas e campilótropas como resultado de um maior desenvolvimento da anti-rafe (figura 8).

A testa diferenciada de Pilocarpus pennatifolius é formada por oito a dez camadas de células. A exotesta é constituída por células amplas, pouco alongadas tangencialmente e com paredes periclinais externas muito espessas e de coloração negra. A mesotesta apresenta células com paredes espessadas, não lignificadas, e a endotesta, à semelhança da exotesta, é constituída por células prismáticas curtas cujas paredes periclinais internas são bastante espessas e também apresentam coloração negra. O exotégmen é representado por células alongadas tangencialmente com espessamentos parietais espiralados ou anelados. O mesotégmen e o endotégmem tornam-se colapsados (figuras 22-24).

As paredes celulares periclinais externas das células da exotesta e as internas da endotesta, espessadas e de coloração escura, parecem conter fitomelano (figura 24). O fitomelano é uma substância de coloração parda ou negra, muito rica em carbono, muito resistente à ação de ácidos e freqüente no pericarpo de espécies de Asteraceae (Compositae) (Font-Quer 1982). Entretanto, para se confirmar essa constatação na semente de Pilocarpus pennatifolius tornam-se necessárias análises químicas mais detalhadas.

Com base na posição e estrutura da principal camada mecânica presente no tegumento, Corner (1976) distingue cinco tipos de tegumento de semente em Rutaceae, com diversas variações menores, porém todos eles sendo exo ou mesotestais. Este autor descreve na semente de Pilocarpus características muito semelhantes às observadas no presente estudo, caracterizando a semente como mesotestal e com tégmen traqueoidal. Boesewinkel (1980) também afirma que na tribo Cusparieae, na qual está incluído Pilocarpus, as sementes apresentam camadas medianas escleróticas na testa, sendo o tégmen total ou 


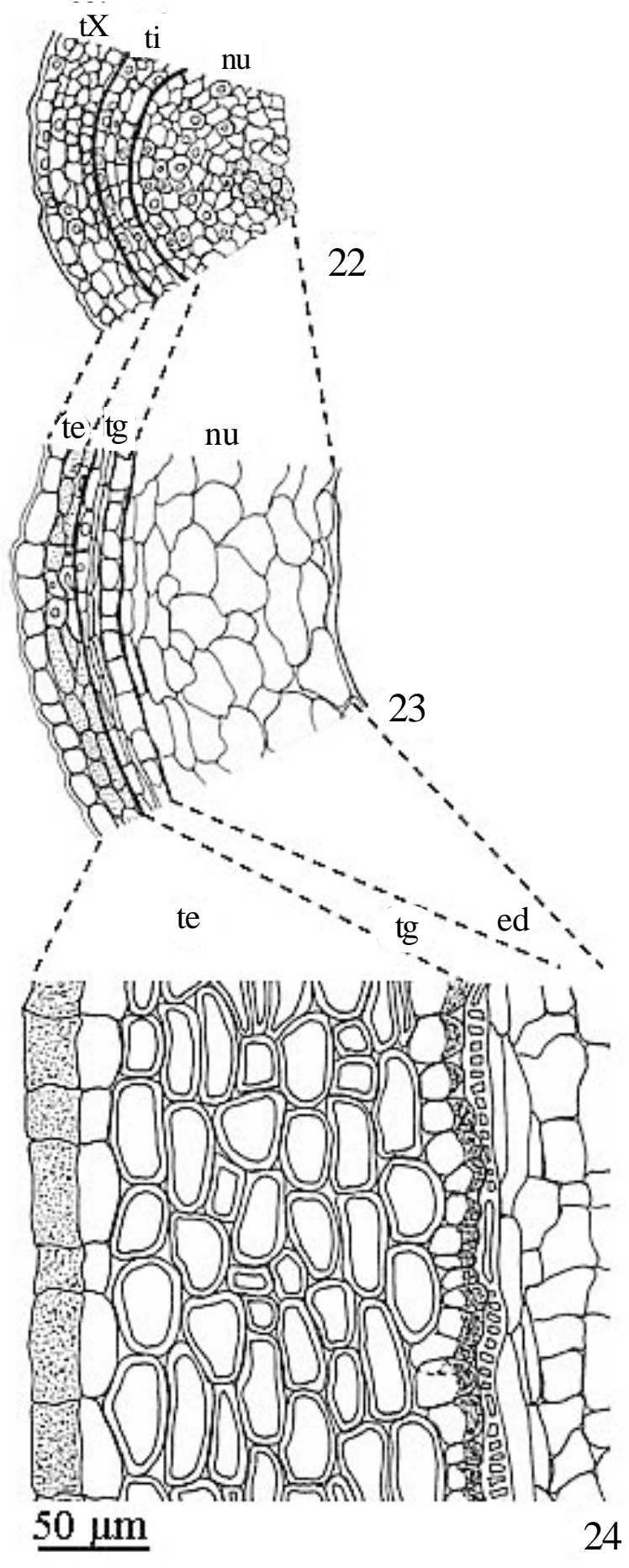

Figuras 22-24. Detalhes de secções transversais do tegumento seminal em desenvolvimento de $P$. pennatifolius. 22 . Óvulo. 23. Semente jovem. 24. Semente madura (ed = endosperma; $\mathrm{nu}=$ nucelo; te = testa; $\operatorname{tg}=$ tégmen $; \mathrm{ti}=$ tegumento interno; tx $=$ tegumento externo).

Figures 22-24. Details of the developing seed-coat in crosssections of $P$. pennatifolius. 22. Ovule. 23. Young seed. 24. Mature seed (ed = endosperm; $\mathrm{nu}=$ nucellus; te = testa; $\operatorname{tg}=$ tegmen; $\mathrm{ti}=$ inner integument; $\mathrm{tx}=$ outer integument $)$. parcialmente traqueoidal. Em Esenbeckia febrifuga, também pertencente a esta tribo, Beltrati (1991) descreve a semente como endotestal e com exotégmen tipicamente traqueoidal.

Em Pilocarpus pennatifolius, a mesotesta é a principal camada mecânica do tegumento, sendo suas células de paredes espessadas, mas não lignificadas, caracterizando-se a semente como mesotestal; o exotégmen apresenta a construção traqueoidal típica da tribo Cusparieae.

O embrião apresenta eixo hipocótilo-radicular curto e cilíndrico e cotilédones plano-convexos carnosos (figura 8), contendo reserva amilácea e oleaginosa. De acordo com a classificação de Martin (1946), o embrião de Pilocarpus pennatifolius é invaginado.

\section{Referências bibliográficas}

BARROSO, G.M., GUIMARÃES, E.F., ICHASO, C.L.F., COSTA, C.G. \& PEIXOTO, A.L. 1984. Sistemática de angiospermas do Brasil. Editora da Universidade de São Paulo e LTC, São Paulo.

BARROSO, G.M., MORIM, M.P., PEIXOTO, A.L. \& ICHASO, C.L.F. 1999. Frutos e sementes: morfologia aplicada à sistemática de dicotiledôneas. Editora da Universidade Federal de Viçosa, Viçosa.

BELTRATI, C.M. 1978. Morfologia e anatomia das sementes e plântulas de Eucalyptus maidenii. Turrialba 28:209-214.

BELTRATI, C.M. 1991. Estudo morfo-anatômico das sementes e plântulas de Esenbeckia febrifuga (St. Hill.) A. Juss. ex Mart. (Rutaceae). Naturalia 16:161-169.

BOESEWINKEL, F.D. 1977. Development of ovule and testa in Rutaceae. I: Ruta, Zanthoxylum, and Skimmia. Acta Botanica Neerlandica 26:193-211.

BOESEWINKEL, F.D. 1978. Development of ovule and testa in Rutaceae. III: Some representatives of the Aurantioidae. Acta Botanica Neerlandica 27:341-354.

BOESEWINKEL, F.D. 1980. Development of ovule and seedcoat in Rutales-Geraniales assembly. University of Amsterdan, Amsterdan, Thesis.

BOESEWINKEL, F.D. \& BOUMAN, F. 1978. Development of ovule and testa in Rutaceae. II: The unigtegmic and pachychalazal seed of Glycosmis cf. arborea (Roxb:) D.C. Acta Botanica Neerlandica 27:69-78.

CRONQUIST, A. 1981. An integrated system of classification of flowering plants. Columbia University Press, New York.

CORNER, E.J.H. 1976. The seed of dicotyledons. Cambridge, University Press, Cambridge.

COWAN, R.S. \& SMITH, L.B. 1973. Rutáceas. In Flora ilustrada catarinense (P.R. Reitz, ed.). Herbário Barbosa Rodrigues, Itajaí. 
FAHN, A. 1990. Plant anatomy. Pergamon Press, Oxford.

FONT-QUER, P. 1982. Diccionario de botánica. Editorial Labor, Barcelona.

GROTH, D., BOARETTO, M.R. \& SILVA, R.N. 1983. Morfologia de sementes, frutos e plantas invasoras de algumas culturas. Revista Brasileira de Sementes 5:151-182.

JENSEN, W.A. 1962. Botanical histochemistry: principles and practice. W.H. Freeman, San Francisco.

JOHANSEN, D.A. 1940. Plant microtechnique. McGraw-Hill Book, New York.

MAHESHWARI, P. 1978. An introduction to the embryology of angiosperms. Tata McGraw-Hill Publishing Company, New Delhi.

MARTIN, A.C. 1946. The comparative internal morphology of seeds. The American Midland Naturalist 36:513-660.

METCALFE, C.R. \& CHALK, L. 1972. Anatomy of the dicotiledons. Clarendon Press, Oxford.

MOURÃO, K.S.M. \& BELTRATI, C.M. 1995. Morfologia dos frutos, sementes e plântulas de Platonia insignis Mart. (Clusiaceae). I. Aspectos anatômicos dos frutos e semente em desenvolvimento. Acta Amazonica 25:11-32.

NETOLITZKY, F. 1926. Anatomie der Angiospermen-Samen. In Handbuch der Pflanzenanatomie (K. Linsbauer, ed.). Gebrüder Borntraeger, Berlin.

O'BRIEN, T.P., FEDER, N. \& MCCULLY, M.E. 1964. Polychromatic staining of plant cell walls by toluidine blue O. Protoplasma 59:368-373.
PIO-CORRÊA, M. 1978. Dicionário das plantas úteis do Brasil e das exóticas cultivadas. Ministério da Agricultura, Rio de Janeiro.

RADFORD, A.E., DICKSON, W.C., MASSEY, J.R. \& BELL, C.R. 1974. Vascular plant systematics. Harper \& Row Publischers, New York.

RAWLINS, T.E. \& TAKAHASHI, W.N. 1952. Technics of plant histochemistry and virology. The National Press, Millbrae.

ROTH, I. 1977. Fruits of angiosperms. In Handbuch der Pflanzenanatomie (K. Linsbauer, ed.) v.10. Gebrüder Borntraeger, Berlin.

SCHMID, R. 1986. On cornerian and other terminology of angiospermus and gymnospermus seed coats: historical perspective and terminological recommendations. Taxon 35:476-491.

SPJUT, R.W. 1994. A systematic treatment of fruit types. Memoirs of the New York Botanical Garden 70:1-182.

SOUZA, L.A. 2003. Morfologia e anatomia vegetal (célula, tecidos, órgãos e plântula). Editora da Universidade Estadual de Ponta Grossa, Ponta Grossa.

SOUZA, L.A., MOURÃO, K.S.M., MOSCHETA, I.S. \& ROSA, S.M. 2003. Morfologia e anatomia da flor e antese de Pilocarpus pennatifolius Lem. (Rutaceae). Revista Brasileira de Botânica 26:175-184.

TILTON, V.R. 1980. Hypostase development in Ornithogalum caudatum (Liliaceae), and notes on the other types of modifications in chalaza of Angiosperm ovules. Canadian Journal of Botany 58:2059-2066. 